\title{
Preoperative Liver Registration for Augmented Monocular Laparoscopy using Backward-Forward Biomechanical Simulation
}

\author{
Erol Özgür • Bongjin Koo • Bertrand Le Roy • \\ Emmanuel Buc · Adrien Bartoli
}

Received: date / Accepted: date

\begin{abstract}
Purpose. Augmented reality for monocular laparoscopy from a preoperative volume such as $\mathrm{CT}$ is achieved in two steps. The first step is to segment the organ in the preoperative volume and reconstruct its 3D model. The second step is to register the preoperative 3D model to an initial intraoperative laparoscopy image. To date, there does not exist an automatic initial registration method to solve the second step for the liver in the de facto operating room conditions of monocular laparoscopy. Existing methods attempt to solve for both deformation and pose simultaneously, leading to nonconvex problems with no optimal solution algorithms.

Methods. We propose in contrast to break the problem down into two parts, solving for $(i)$ deformation and (ii) pose. Part (i) simulates biomechanical deformations from the preoperative to the intraoperative state to predict the liver's unknown intraoperative shape by modeling gravity, the abdominopelvic cavity's pressure and boundary conditions. Part (ii) rigidly registers the simulated shape to the laparoscopy image using contour cues.

Results. Our formulation leads to a well-posed problem, contrary to existing methods. This is because it exploits strong environment priors to complement the weak laparoscopic visual cues.

Conclusions. Quantitative results with in-silico and phantom experiments and qualitative results with laparosurgery images for two patients show that our method outperforms the state-of-the art in accuracy and registration time.
\end{abstract}

Keywords liver registration · monocular laparoscopy $\cdot$ biomechanical simulation

\section{Introduction}

Context. Augmented reality (AR) is a promising guidance system for monocular liver laparoscopy. However it requires an initial liver registration, for which there has been so far no automatic solution in monocular laparoscopy. We address this initial liver registration problem, which is more precisely to register a patient's preoperative 3D liver model to an intraoperative laparoscopy image. The term 'initial' is because the registration could be updated in a later stage to cope with laparoscope motion. We use MITK's region-growing

EnCoV, IP, UMR 6602 CNRS, Université Clermont Auvergne, SIGMA, France

\{erol.ozgur, bongjin.koo\}@uca.fr, \{bleroy, ebuc\}@chu-clermontferrand.fr, adrien.bartoli@gmail.com 
tool [1] to segment and reconstruct the 3D liver model from the patient's preoperative CT volume. The 3D model contains the key anatomical parts such as the tumors and veins, necessary for AR guidance.

The registration problem. Initial liver registration in monocular laparoscopy is extremely difficult to solve. This is because the monocular laparoscope does not provide depth and thus the registration has to be a multimodal and volume-to-image one. Furthermore, the liver is deformed (due to gravity and the pneumoperitoneum) and only partially visible in the laparoscopy image. This implies that the visual cues constrain the registration only locally. Therefore, formulating registration based on only the visual cues from a laparoscopy image is likely to be ill-posed and to have multiple weakly constrained solutions.

Existing work and remaining challenges. The literature in image-guided surgery is substantial. We therefore focus our review on the initial registration problem in monocular laparoscopy. Several methods have been proposed to solve initial liver registration. Unfortunately, none of them works in de facto conditions of a monocular laparoscopy procedure $[2,3]$ but two recent semi-automatic methods $[4,5]$. However, the methods $[4,5]$ still leave room for improvement. The method [4] takes neither gravity load nor the pneumoperitoneum pressure into account in registration. It computes registration using only the weak laparoscopy visual cues, which consequently degrades progressively in unseen parts of the liver. The method [5] takes only the pneumoperitoneum pressure into account in registration and requires a very good initialization to avoid local minima. From a gravity compensation viewpoint, all existing methods using a biomechanical model for registration in laparoscopy ignore the gravity load and use the biomechanical model reconstructed from a segmented preoperative CT as an undeformed and stressless reference model. Because this model is in fact gravity-loaded, running a laparoscopic pneumoperitoneum simulation with it as the reference model [5-8] generates inaccuracies. Only [9] compensates for the gravity load by reversing its direction before applying laparoscopic pneumoperitoneum simulation for surgical planning purposes. However [9] does not solve registration. From an optimization viewpoint, existing methods for the liver solve the initial deformable registration problem by optimizing for both deformation and pose in a single step using only the weak laparoscopy visual cues $[4,5]$. This yields nonconvex problems, which cannot be solved unless a very good initialization is provided. From an automation viewpoint, this initialization requirement and also the laparoscopy image processing (i.e., detection and recognition of anatomical landmarks and contours) make the current registration methods very unlikely to be automated in monocular laparoscopy.

There exist advanced methods which solve the initial registration automatically but were designed to work in different conditions from monocular liver laparoscopy. The method [10] requires multiple intraoperative images of a fully visible and rigid organ for rigid registration using the silhouette; the method [11] requires an intraoperative CT scan for rigid registration using shading; the method [12] requires multiple cameras for grabbing rigid views of the organ to build a silhouette-based visual hull for deformable registration, and provides results only for simulated data. We remark that depth-based methods using stereo such as $[13,14]$ form a vast part of the literature. Knowing depth greatly simplifies registration, as can be seen in $[13,14]$ and references therein. However, stereo is not commonly available in the current surgical theatres and hence we do not consider it available.

One of the current challenges in monocular liver laparoscopy is to exploit both the environment priors (gravity and the pneumoperitoneum) and the visual cues to improve registration. 
Proposed solution and experimental validation. We propose a semi-automatic solution to the initial liver registration problem. Its inputs are the liver's preoperative 3D model and a single laparoscopy image. It thus complies well with monocular laparoscopy and exploits both the environment priors and visual cues. We formulate the deformable registration problem as two consecutive subproblems, solving for $(i)$ deformation and (ii) pose. Both subproblems are well-posed and well-constrained. We solve subproblem $(i)$ by simulating the biomechanical deformations between the preoperative and intraoperative states to predict the liver's unknown initial intraoperative shape. This requires us to simulate first the gravityless shape from the gravity-loaded preoperative 3D model in supine orientation and then the shape under pneumoperitoneum pressure and gravity-load in a reverse trendelenburg orientation. We then solve subproblem (ii) by registering rigidly the simulated shape to the input laparoscopy image using contour cues. These visual cues combine the liver's silhouette and a few curvilinear anatomical landmarks. They are marked on the input laparoscopy image by the user. Their corresponding location on the preoperative 3D model is then assigned automatically. The proposed solution is validated quantitatively with in-silico and phantom experiments and qualitatively with in-vivo laparoscopy images.

\section{Methodology}

\subsection{Overview}

We show in Figure 1 the pipeline of the proposed initial registration solution, whose six main stages are as follows. (i) We segment the liver and its surrounding structures such as the abdominal wall and the scanning table in the CT volume. The segmented liver forms a gravity-loaded shape. This shape defines a connected subset of $\mathbb{R}^{3}$ which we tessellate and represent as a tetrahedral mesh. Following [15], each vertex is considered a particle with an associated mass. The liver's mass is computed by multiplying its volume with the human liver density $\rho=1020 \mathrm{~kg} / \mathrm{m}^{3}$ [16]. (ii) We compute the gravity direction from the scanning table's surface normal. (iii) We simulate backward to remove the gravity deformation from the segmented liver shape. (iv) We simulate forward to exert pneumoperitoneum pressure and gravity in reverse trendelenburg orientation. $(v)$ We grab a maximally zoomed-out laparoscopy image and mark the liver contours. (vi) Finally, we compute the pose and augment the laparoscopy image.

It is critical to minimize the amount of time required in the OR and precompute as much results as possible, since before surgery computation time is virtually unlimited. Stages $(i)$ to (iii) are obviously done in the preoperative step. However, in stage (iv) the reverse trendelenburg orientation required to define the gravity direction only becomes available at the time of surgery. Nonetheless, we know that it covers a range from $0^{\circ}$ to $30^{\circ}$. We therefore propose to accelerate the registration by also solving $(i v)$ in the preoperative step by varying the gravity direction with a $1^{\circ}$ step and storing all results. This step size is reasonable due to the $\pm 0.3^{\circ}$ uncertainty in angle measurement with a smartphone [17] and a small uncertainty that might exist on the patient's pose on a cushion-covered surgery table. Only stages $(v)$ and (vi) need to be solved in the intraoperative step. In stage (v), we do not need to compensate for the laparoscope movement, the breathing and the heartbeat, since the liver's contours are marked on a single grabbed image. The pose computation in stage (vi) uses the shape retrieved from the stored set of precomputed simulated shapes, using the smartphone's angle measurement. 


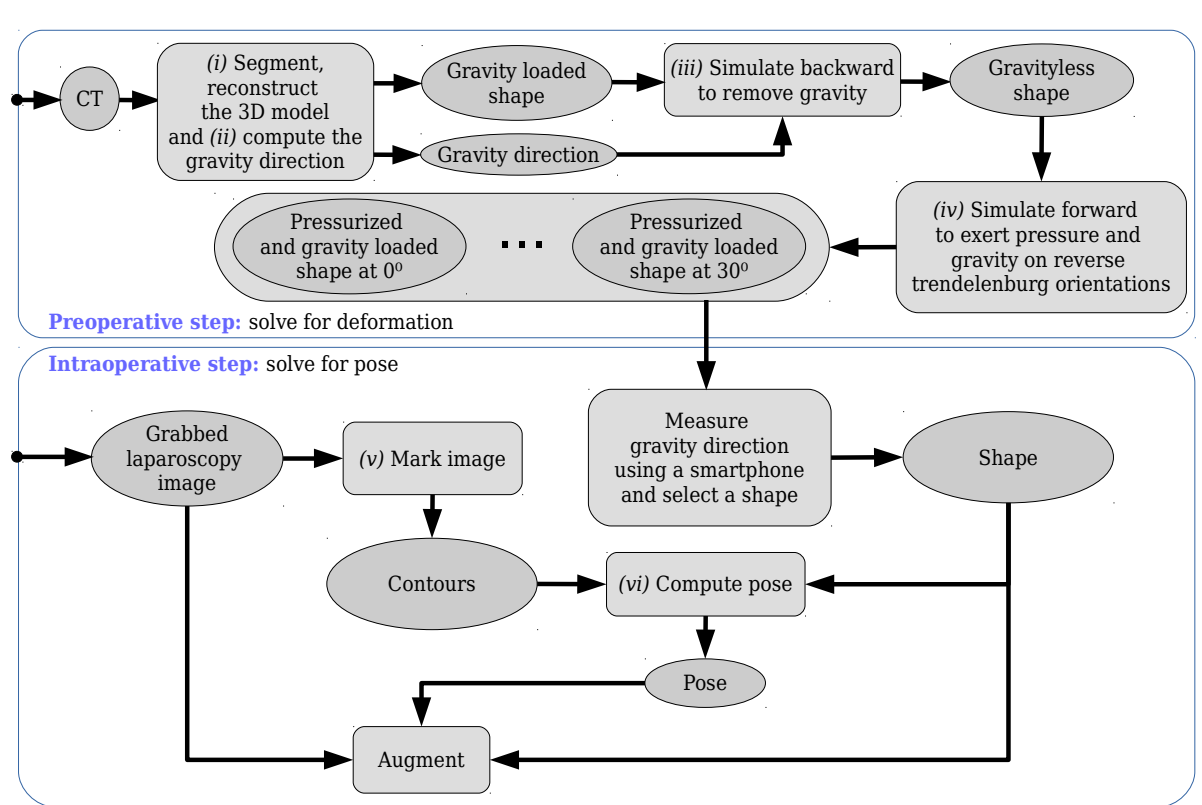

Fig. 1 Pipeline of the proposed initial liver registration and augmentation solution.

\subsection{Backward-Forward Biomechanical Simulations}

We describe our solution to stages (iii) and (iv) of the pipeline, corresponding to gravityless shape simulation and shape under gravity and pressure simulation, respectively.

\subsubsection{Gravityless Shape Simulation}

Proposed solution. Gravityless shape simulation is a backward simulation problem. It can be solved by either analytical methods designed for a specific biomechanical model [18] or fixed-point iterative algorithms using forward simulations [19-22]. Both ways yield similar solutions [23]. We adopt a fixed-point algorithm because it handles biomechanical deformation models generically (Algorithm 1). The principle is to initialize the shape, simulate forward from it, and update the shape by comparing with the measured, gravity-loaded shape. We use the Neo-Hookean model. We set the human liver's mechanical parameters as $60 \mathrm{kPa}$ for Young's modulus and 0.49 for Poisson's ratio. In Algorithm 1, line 02 performs a forward deformation simulation from the gravityless shape estimate using the known gravity forces and the boundary conditions. Forward deformation simulation is a solved problem. Therefore any FEM library can be used to implement the Simulate Deformation function. We use our own implementation of [15,24]. Then, in Algorithm 1, lines 03 and 04 use the shape discrepancy $\triangle \mathcal{M}$ to update the gravityless shape estimate. Finally, the algorithm stops when either a shape from the forward simulation converges to the gravity-loaded shape or the maximum number of iterations is reached. Convergence is assessed using the root mean squared (RMS) value of the shape discrepancy.

Boundary conditions. As boundary conditions, we constrain the depth of a set of points. The depth-constrained points are allowed to move in any direction but not deeper than where they initially were along the gravity direction. The depth-constrained points are chosen on the gravity-loaded preoperative 3D model's surface below a level and form a bathtub shape to 


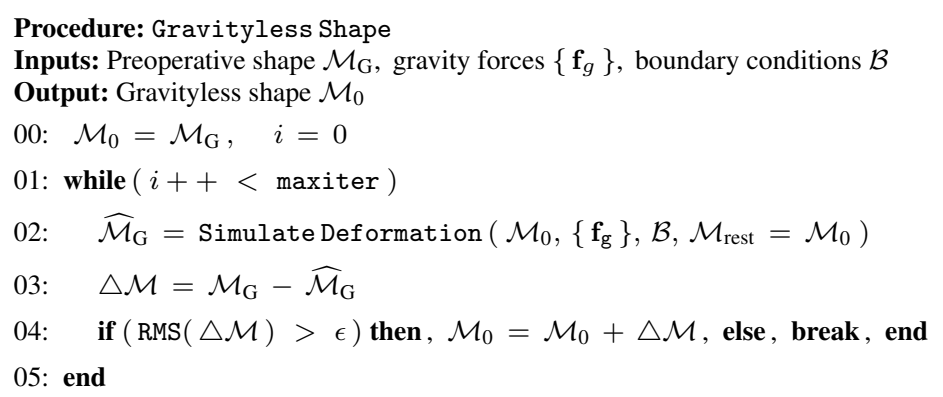

Algorithm 1: Fixed-point iterative algorithm for estimating the gravityless shape. We use the boundary conditions $\mathcal{B}=$ \{depth constraints $\}$, a precision threshold $\epsilon=10^{-3} \mathrm{~m}$ and a maximum number of iterations maxiter $=10 . \mathcal{M}_{\text {rest }}$ represents the reference unstressed shape for the forward deformation simulation.

prevent the model falling freely under the forward gravity simulation. This gives the model enough flexibility to reach its gravityless shape. This is the shape that would be naturally taken by the liver in an unconstrained environment. Figure 2 shows an example.
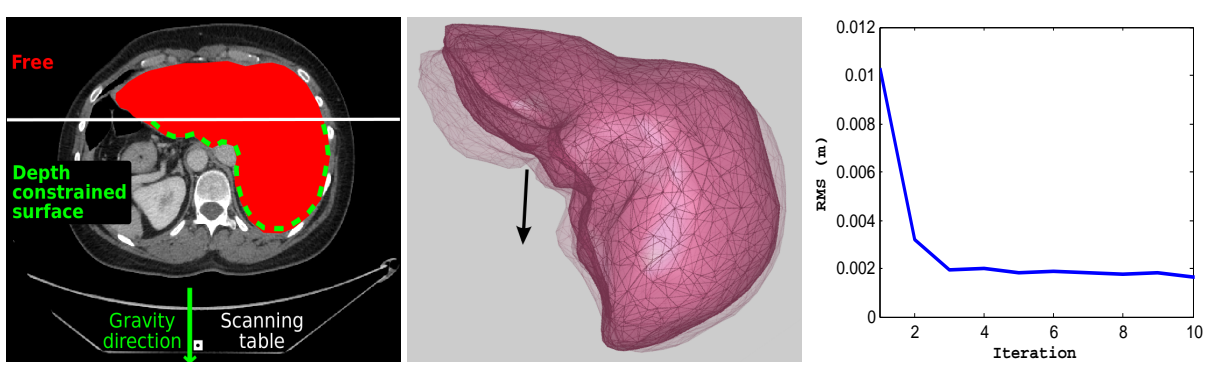

Fig. 2 The left image shows an axial CT slice (viewed from head-to-feet) with the segmented liver (red) and its depth-constrained surface (dashed green) below the horizontal white line, and the gravity direction (green arrow orthogonal to the scanning table's flat surface). The middle image shows the simulated gravityless shape (red opaque) viewed over the gravity-loaded shape (transparent) and the gravity direction (arrow). The right graph shows the evolution of $\operatorname{RMS}(\triangle \mathcal{M})$ versus the iteration of Algorithm 1 . The gravityless liver shape seen in this figure corresponds to patient $-A$ in $\S 4.3$.

\subsubsection{Shape under Gravity and Gas Pressure Simulation}

Proposed solution. Shape prediction under gravity and gas pressure is a forward simulation problem. It is solved in the preoperative step for a range of orientations able to express the possible surgical setups (Algorithm 2). The abdominal cavity is initially pressurized to $15 \mathrm{mmHg}$ for safe trocar placement. The pressure is then lowered to the standard operating value of $12 \mathrm{mmHg}$. In Algorithm 2, line 02 computes the particles' pressure forces for the current shape estimate of the liver and line 03 performs one time-step of forward deformation simulation using the given forces and boundary conditions. As in the backward simulation of $\S 2.2 .1$, we use our own implementation of [15,24] for the Simulate Deformation For One Time Step function. If the simulation has not converged, 
line 05 updates the liver shape. Finally, Algorithm 2 stops when either the simulation converges or the maximum number of iterations is reached.

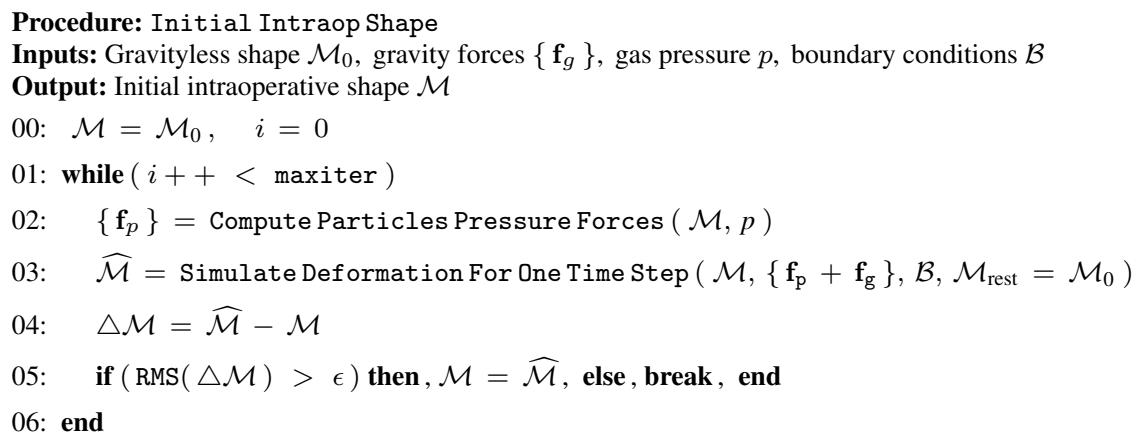

Algorithm 2: Algorithm for estimating the initial intraoperative shape with gas pressure $p=12 \mathrm{mmHg}$ and boundary conditions $\mathcal{B}=\{$ plane constraint $\}$. We use a precision threshold $\epsilon=10^{-6} \mathrm{~m}$ and a maximum number of iterations maxiter $=10^{3} . \mathcal{M}_{\text {rest }}$ represents the reference unstressed shape for the forward deformation simulation.

Boundary conditions. The abdominal cavity packs the soft organs compactly and allows them to slide past each other. In the cavity, the liver's lower visceral surface sits perfectly like a hat on top of the other organs. We have observed that, in a pressurized abdominal cavity, after the liver is separated from its falciform ligament, it gains enough mobility to float over the other organs. In order to observe and understand this phenomenon, we respectively pressurized, de-pressurized and re-pressurized the abdominal cavity between about $5 \mathrm{mmHg}$ and $12 \mathrm{mmHg}$. This generated a tide-like motion of the liver within a few centimeters, as can be seen in Figure 3. Therefore, as boundary conditions, we let the simulated liver freely deform and slide on a plane. This plane represents roughly the contact surface between the liver and the other organs. We obtain the plane as the best fit to the visceral contact surface of the gravityless preoperative 3D liver model.

\subsection{D Pose from 2D Contours Computation}

We describe our solution to stage (vi) in the pipeline. Matching points automatically between the textureless preoperative 3D model and an input laparoscopy image is an unsolved problem. Consequently, we face a $3 \mathrm{D}$ pose computation problem from a single 2D image in the absence of point correspondences. However, we can count on liver contour correspondences (the silhouette and the ridge and falciform ligament contours) which can be quickly established semi-automatically between the image and the preoperative 3D model following [4]. We then run an iterative closest point (ICP) algorithm to solve the 3D pose of the model from these correspondences (Algorithm 3). In Algorithm 3, line 02 computes the $3 \mathrm{D}$ model contours corresponding to the marked image contours; line 03 projects the 3D model contours onto the image; line 04 computes the closest 3D-2D point pairs between the projected 3D model contours and their corresponding marked image contours; line 05 


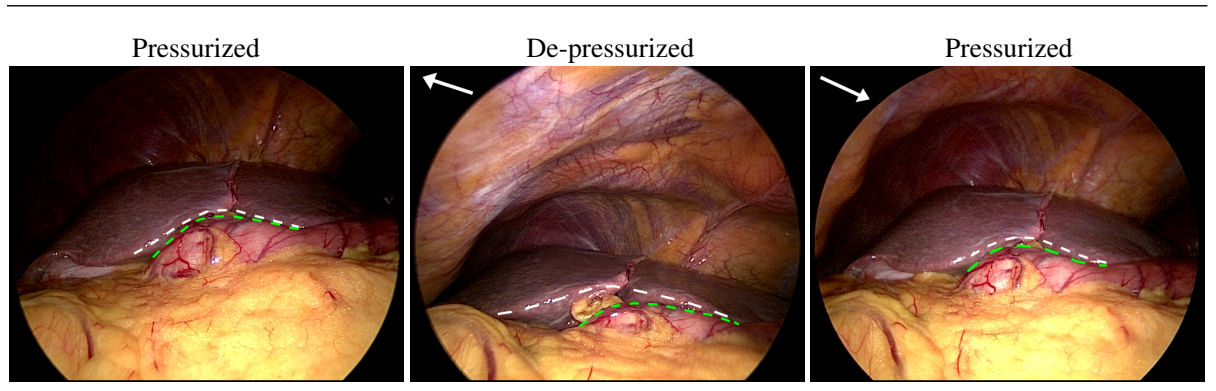

Fig. 3 Liver deformation at a $12 \mathrm{mmHg}$ pressure (left), at about $5 \mathrm{mmHg}$ (middle) and again at a $12 \mathrm{mmHg}$ pressure (right). We observed that the liver floats up (middle) or sinks down (right) up to a few centimeters with respect to its surrounding structures. We marked the same piece of contours on the liver (white dashed) and the stomach (green dashed) for each image to ease the observation of displacements. The arrows show the liver's displacement direction.

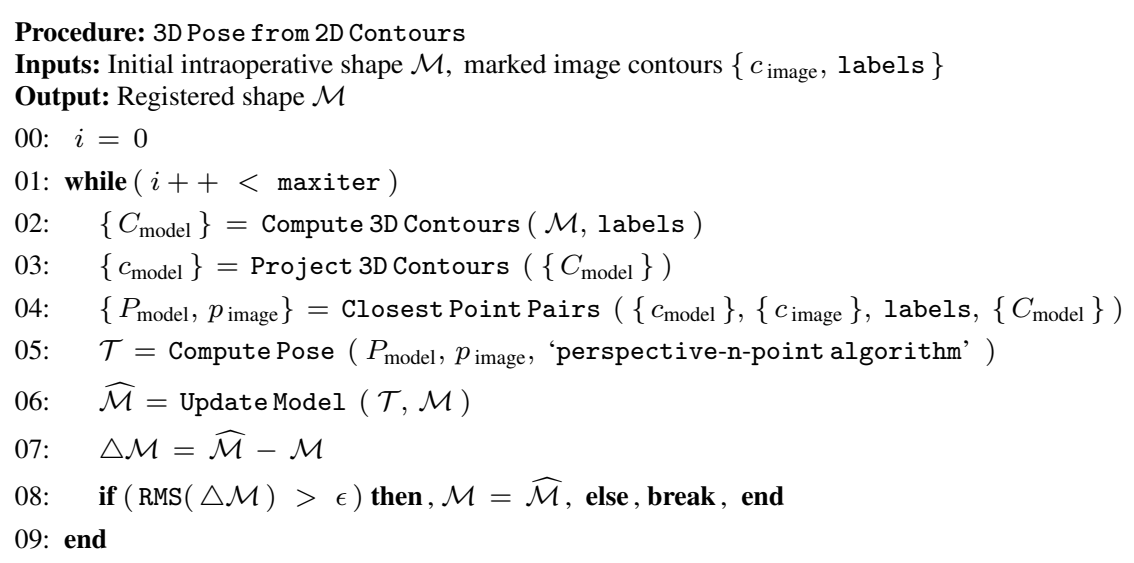

Algorithm 3: Pose computation from 3D-to-2D contour correspondences. We use a precision threshold $\epsilon=10^{-6} \mathrm{~m}$ and a maximum number of iterations maxiter $=20$. A label $\in$ \{silhouette, ridge, falciform ligament $\}$ indicates the type of a marked curve.

computes the best pose between these $3 \mathrm{D}-2 \mathrm{D}$ point pairs using the perspective- $n$-point algorithm [25]; and in lines 01 to 09 , the while loop iterates until the pose converges or the maximum number of iterations is reached.

\section{Experiments}

\subsection{Compared Methods}

We compare our method with deformable [4] and rigid [2] registration, referred to as deformable and rigid, respectively. Both deformable and rigid use directly the segmented preoperative $3 \mathrm{D}$ model in registration and thus do not exploit environment priors. 


\subsection{Validation}

We provide the following experiments to validate the proposed initial liver registration method: (i) quantitative in-silico experiments using patient- $A$ 's segmented liver model from their preoperative CT scan, (ii) quantitative phantom experiments, and (iii) qualitative invivo experiments with patients $A$ and $B$ 's laparosurgery images.

\subsubsection{Quantitative In-silico Experiments}

Setup. Regardless of how different the compared registration methods are, there exist two important factors which may directly affect their accuracy: the initialization of the preoperative 3D liver model and the visibility of the patient's liver in the laparoscopy image. We therefore conducted two sets of quantitative simulations to evaluate these. First, we evaluated the convergence of the registration algorithms under seven different initial poses of the preoperative 3D model while the visibility of the contours was kept fixed. Second, we evaluated the convergence of the registration algorithms under four different visibility levels of the contours while the initial pose of the preoperative 3D model was kept fixed. To be able to evaluate the registration quantitatively, we assumed ideal conditions where the patient's liver model is deformed only under the known gravitational force, pneumoperitoneum pressure at $12 \mathrm{mmHg}$ and the boundary conditions. We used these deformed shapes as ground-truth and rendered their synthetic images to form the input laparoscopy images. Registration accuracies were expressed as RMS of the errors (RMSE) between the corresponding points of the ground-truth shape and the registered shapes. We also remark that in quantitative simulations deformable does not exploit the approximate end points of the ridge contour as correspondences.

Registration accuracy versus pose initialization. An insufflated abdominal cavity forms an encapsulated space for the liver. In this space, an approximate automatic initialization for the liver pose can be computed from the preoperative radiological data as in [4]. In this automatic initialization, it is assumed that the laparoscope's keyhole is located near the belly button and the laparoscope is directed at the patient's liver. Consequently, the liver is located approximately in the center of the laparoscope's field of view. This field of view's major radius does in practice not exceed $10 \mathrm{~cm}$ and the automatic initialization falls easily into it. We therefore progressively increased the displacement perturbations from the ground-truth with a step of $1 \%$ magnitude and up to $7 \%$, and evaluated convergence. Results are presented in Figure 4. A $1 \%$ magnitude perturbation in Figure 4 corresponds to a $1 \mathrm{~cm}$ translation and $10^{\circ}$ of rotation about a random axis.

Registration accuracy versus contour visibility. A piece of the liver's silhouette is always visible on the upper convex diaphragmatic surface while the rest is usually occluded by the other organs and fat. A piece of the liver's ridge contour between its convex and concave parts is always visible on the lower part while the rest is usually occluded by the liver itself, other organs and fat. The falciform ligament might be partially visible or not visible at all. One can observe these instances in Figures 3 and 8. We therefore started convergence simulations with an initial piece of silhouette (less than half of the full silhouette) from the upper convex surface and an initial piece of ridge contour (less than half of the full ridge perimeter) from the lower part. We then progressively decreased the visibility level of these pieces of contour and evaluated convergence performance. Results are presented in Figure 5. 


\subsubsection{Quantitative Phantom Experiments}

We simulated the deformation induced by the pneumoperitoneum pressure on a patient's gravityless preoperative 3D liver model. We then 3D printed out the mold of the deformed $3 \mathrm{D}$ liver model to eventually create its silicone phantom. We placed the phantom in a different pose compared to preoperative CT scanning, in order to obtain a realistic gravity-induced deformation, similarly to the reverse trendelenburg position. The resulting ground-truth shape was then acquired by structured-light 3D scanning using a turntable (DAVID SLS3 3D Scanner). The ground-truth shape thus encodes both gravity and pneumoperitoneum deformation. Figure 6 shows the steps of the phantom dataset preparation. The ground-truth shape's top part has less visibility in the camera and the bottom part was mostly occluded during scanning. Therefore, it has two holes, one on the top and one at the bottom. Figure 7 shows the registration results of the compared methods.

\subsubsection{Qualitative In-vivo Experiments}

We compared our registration solution to existing methods qualitatively for in-vivo laparosurgery images of two patients, $A$ and $B$. The laparoscopes used for patients $A$ and $B$ were calibrated using images of a checkerboard and Agisoft's Lens software. The laparoscopes' focal lengths were 992 pixels for patient- $A$ and 932 pixels for patient- $B$.

Patient- $A$. Figure 8 shows the segmented model from the CT volume, an input image and a control image of patient- $A$ for qualitative comparison of the registrations. The control image shows the liver with the same deformation but from a different viewpoint. The left-most half of the control image reveals a part of the liver which is unseen in the input image. Patient- $A$ was aligned at a $20^{\circ}$ reverse trendelenburg orientation and undergoing a right hepatectomy. Results of patient- $A$ are presented in Figure 9.

Patient- $B$. Figure 10 shows the segmented model from the CT volume, an input image and a control image of patient- $B$. Patient- $B$ was aligned at a $15^{\circ}$ reverse trendelenburg orientation and undergoing a segmentectomy 6 . For patient- $B$, we did not have a control image with the same liver deformation but a similar deformation from a different viewpoint. The slight difference in deformation is due to an instrument lifting the liver in the lower part of the control image. Results of patient- $B$ are presented in Figure 11.

\subsection{Registration Uncertainty}

We provide an optimistic estimate, and thus a lower bound, on the registration uncertainty. In our experiments, the quantifiable uncertainties arise from three main sources: $(i)$ the voxel size in the CT volume, (ii) the technique used for liver segmentation and 3D model reconstruction from the CT volume and (iii) the imprecision of contour localization in the laparoscopy image. We now quantify the uncertainties of these sources. A typical CT voxel size used in preoperative scanning is $0.5 \mathrm{~mm} \times 0.5 \mathrm{~mm} \times 1 \mathrm{~mm}$. This introduces a positional uncertainty of approximately $u_{i} \approx \pm 1.2 \mathrm{~mm}$. We assume that there exists a systematic error of $1 \%$ in the volume segmentation and 3D model reconstruction techniques for the liver shape. This introduces a positional uncertainty of approximately $u_{i i} \approx \pm 2.5 \mathrm{~mm}$, considering that the average dimensions of an adult human liver is $10 \mathrm{~cm}$ 
$\times 15 \mathrm{~cm} \times 20 \mathrm{~cm}$. We assume that there exists a systematic error of $1 \%$ in contour localization in the typical laparoscopic surgery setting. This introduces a positional uncertainty of approximately $u_{i i i} \approx \pm 2 \mathrm{~mm}$, considering a maximally zoomed-out laparoscope whose focal length is around 1000 pixels, image size is $1920 \times 1080$ pixels, and visualizing the liver at a distance of approximately $10 \mathrm{~cm}$. Finally, adding these uncertainties in quadrature yields approximately $u_{q} \approx \pm 3.5 \mathrm{~mm}$ uncertainty on the registration accuracy, where $u_{q}=\sqrt{u_{i}^{2}+u_{i i}^{2}+u_{i i i}^{2}}$. We remark that there also exist other sources of uncertainties. These are due to the imprecisions of gravity direction measurement, the elastic model (homogeneous tissue, isotropic behavior, generic parameter values) used to simulate the liver's deformation and the boundary conditions.

\section{Results}

4.1 Quantitative Results for the In-silico Experiments

Registration accuracy versus pose initialization. We observe in Figure 4 that all methods are rather insensitive to initialization within the laparoscope's field of view, and that ours is consistently and substantially more accurate.
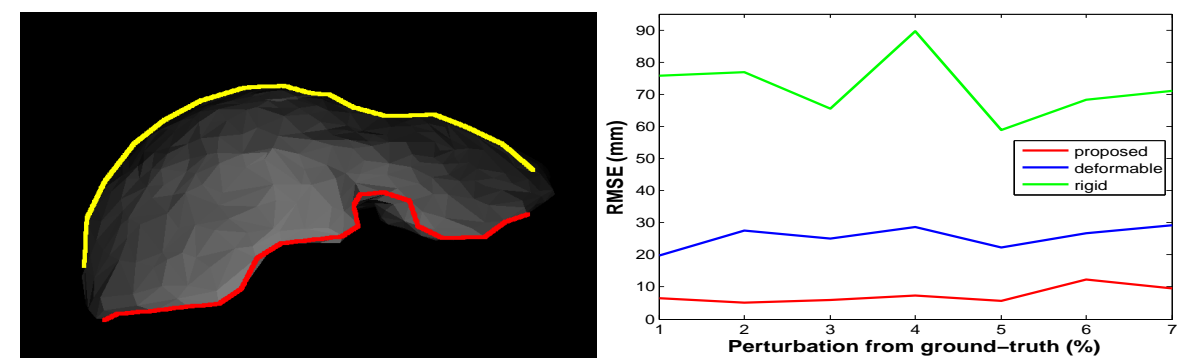

Fig. 4 Quantitative comparison of registration. The left image shows the ground-truth shape with the marked contours. The right graph shows the RMSE versus initial pose perturbation.

Registration accuracy versus contour visibility. We observe in Figure 5 that the accuracy of all methods gently degrades with an increasing level of occlusion and that ours remains more accurate.
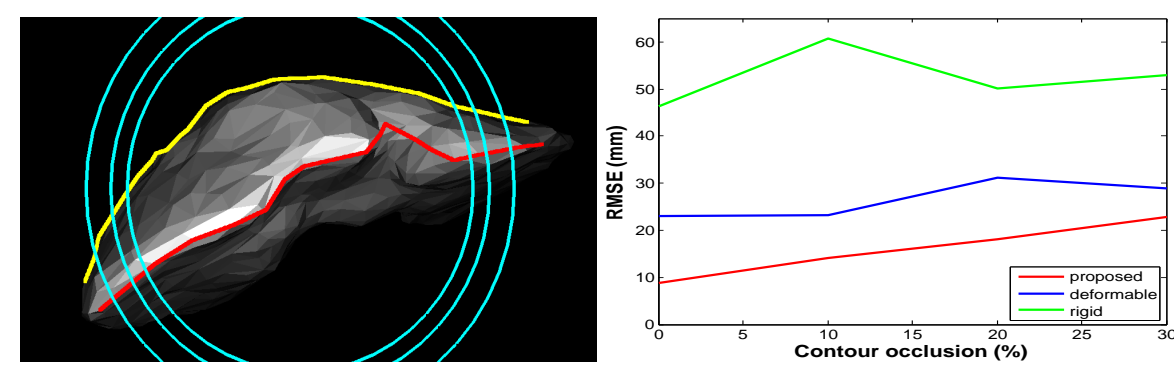

Fig. 5 Quantitative comparison of registration. The left image shows the ground-truth shape with the marked contours and their visibility field of view circles. From the largest to the smallest, the field of view circles occlude approximately $10 \%, 20 \%$ and $30 \%$ of the marked contours, respectively. The right graph shows the RMSE versus contour occlusion level. 
Registration time. We report the computation time for the main loop of each method. Both rigid and the proposed registration methods take about 45 seconds on average, while deformable [4] takes about 150 seconds on average.

\subsection{Quantitative Results for the Phantom Experiments}

We observe in Figure 7 that the proposed method registers better on the lower part of phantom on the input and it has lower 3D errors than deformable and rigid. The proposed method's minimum, maximum and average 3D errors are $0.1 \mathrm{~mm}, 33.6 \mathrm{~mm}$ and $11.2 \mathrm{~mm}$ (stdv. $5.7 \mathrm{~mm}$ ), respectively. deformable's minimum, maximum and average 3D errors are $0 \mathrm{~mm}, 38.6 \mathrm{~mm}$ and $13.4 \mathrm{~mm}$ (stdv. $7.1 \mathrm{~mm}$ ), respectively. rigid's minimum, maximum and average 3D errors are $0.1 \mathrm{~mm}, 43.2 \mathrm{~mm}$ and $18.9 \mathrm{~mm}$ (stdv. $9.3 \mathrm{~mm}$ ), respectively. We also observe in Figure 7 that the depth error for all the methods increases away from the phantom's silhouette. This is explained by the fact that the silhouette gives a strong registration constraint, whose influence naturally decreases with an increasing distance to it. Though all the methods present this common trend, ours remains consistently more accurate.

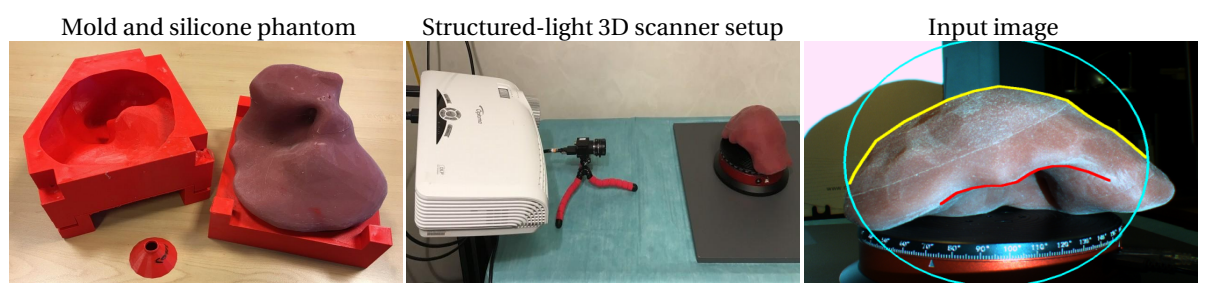

Fig. 6 The silicone phantom's mold, scanning setup and an input image with marked contours for testing the compared methods.

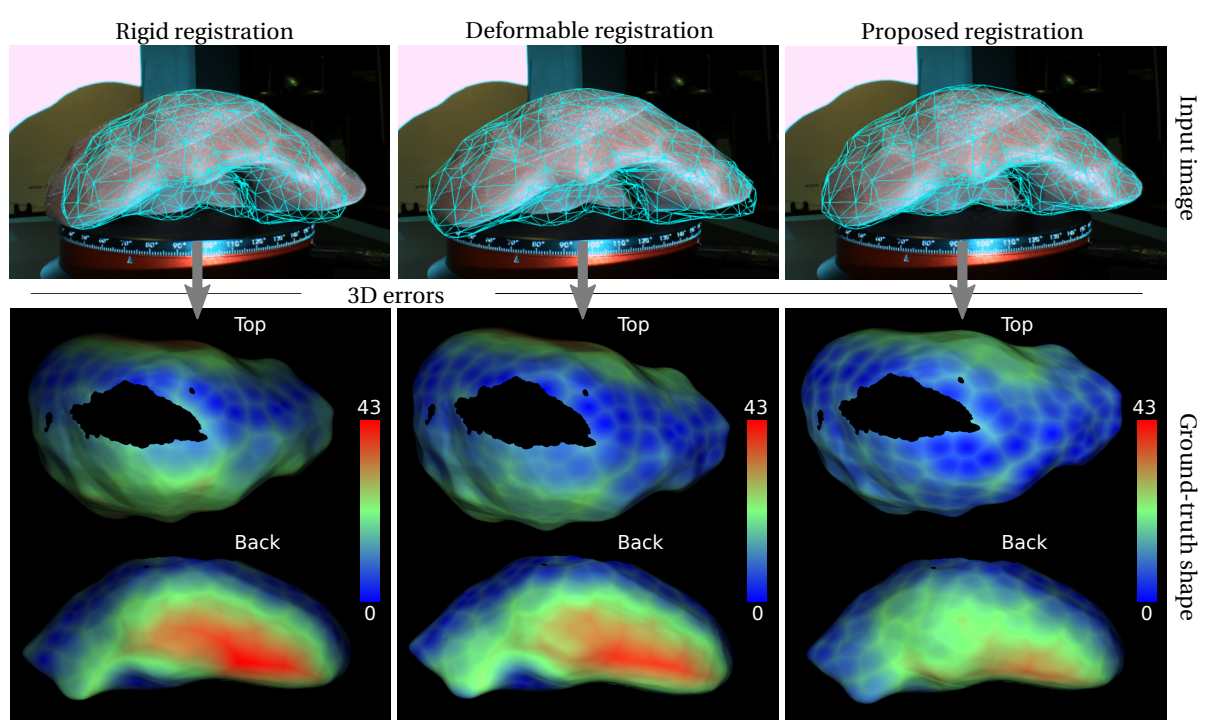

Fig. 7 The first row shows the registration for the compared methods. The second row shows the colormapped 3D errors $(\mathrm{mm})$ from different viewpoints on the ground-truth shape measured with respect to the registered shapes in terms of the closest point distances. 
4.3 Qualitative Results for Laparosurgery Images

Patient- $A$. In the first row of Figure 9, we observe that the proposed registration and deformable match the observation well. On the other hand, in the second row, we observe that the proposed registration covers substantially better the deformation of the unseen part. This is because deformable optimizes the deformation using only the input image, which contains no information about the unseen part and therefore constrains the shape weakly and locally. In contrast, ours optimizes the deformation using the strong environment priors (pneumoperitoneum pressure and gravity) which constrain the shape globally. In this case, rigid was significantly worse.
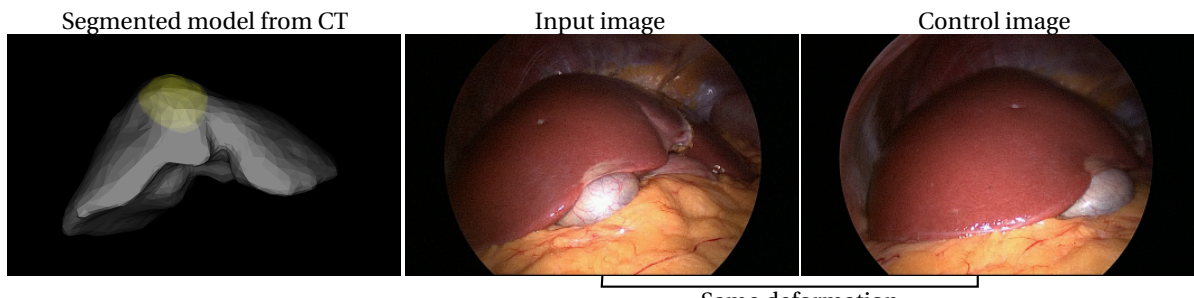

Same deformation

Fig. 8 Patient- $A$. The segmented liver model with the tumor (yellow) from the CT volume, an input image and a control image for qualitative comparison of the registrations. The control image shows the liver with the same deformation from a different viewpoint.

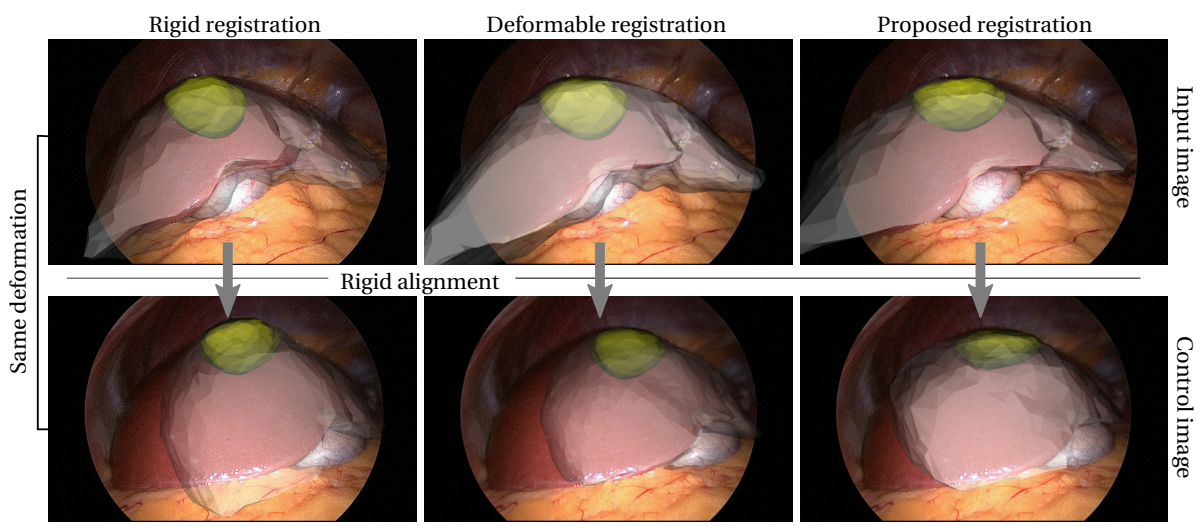

Fig. 9 Patient- $A$. Qualitative comparison of in-vivo registrations for the images seen in Figure 8. On the input image, both deformable and the proposed registration look convincing. In the second row, we check the quality of the registered shapes by rigidly aligning them to the control image.

Patient-B. Figure 11 shows in the first row the results of rigid, deformable and the proposed method on the input image and in the second row the rigid alignment of the registered shapes on the control image. On the input image, we see that both deformable and the proposed registration match the observed shape to a good extent, which are satisfying results. rigid matches worse than the others in the rightmost side of the input image. On the control image, it is difficult to tell which method is better than the other. Nonetheless, the 
proposed registration's shape matches well overall, except for the lower part of the image where the slight deformation occurred.
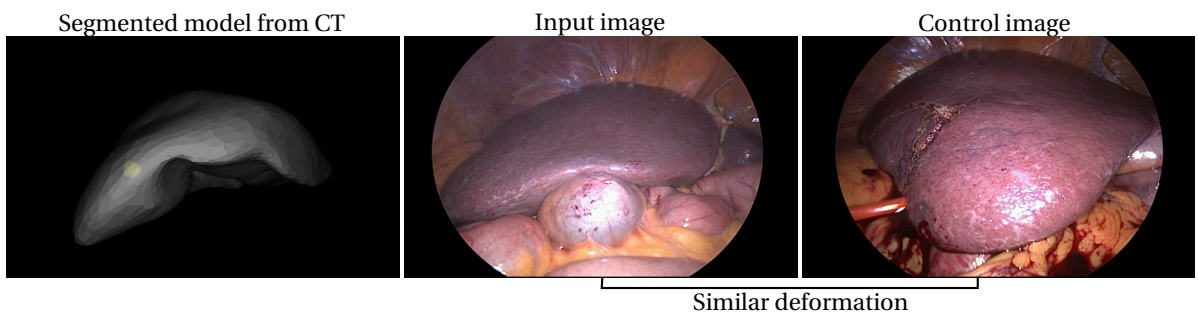

Fig. 10 Patient- $B$. The segmented liver model with the tumor (yellow) from the CT volume, an input image and a control image for qualitative comparison of the registrations. The control image shows the liver with a similar deformation from a different viewpoint.

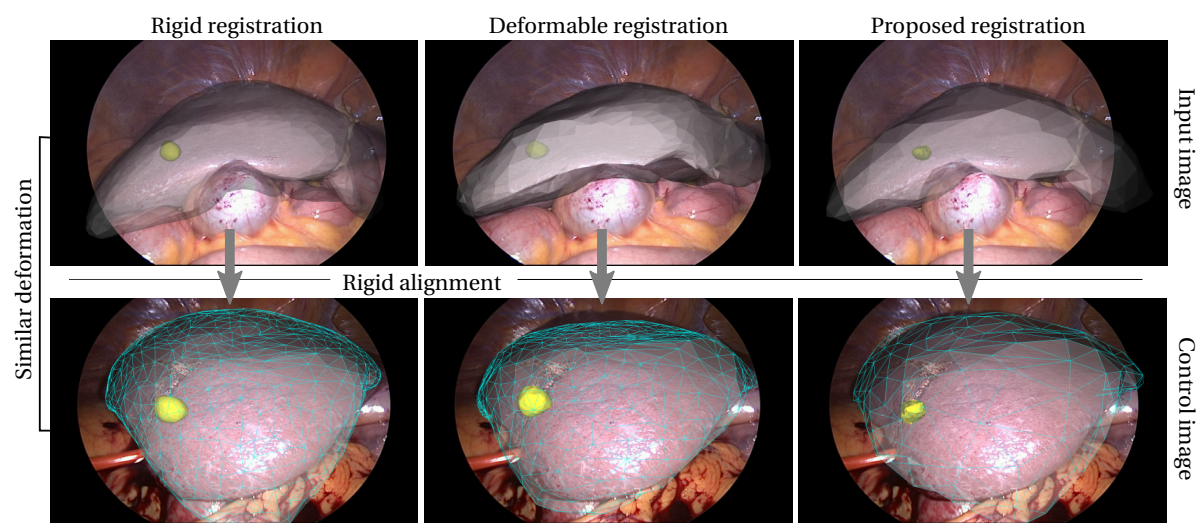

Fig. 11 Patient- $B$. Qualitative comparison of in-vivo registrations for the images seen in Figure 10. On the input image, both deformable and the proposed registration look convincing. In the second row, we check the quality of the registered shapes by rigidly aligning them to the control image.

\section{Discussion}

About the boundary conditions. The accuracy of the proposed solution depends on the boundary conditions used in the forward simulation given in Algorithm 2. The identification of these boundary conditions in liver deformation simulation is very difficult [26] and still forms an open problem. The difficulty comes from the imprecise localization of the liver's ligament connections in the preoperative CT images, the ligaments' unknown soft behavior and the liver's unknown mobility space. Furthermore, all these are patient-specific. However, we showed that the proposed solution using even a simple approximation of these conditions has the potential to beat the state-of-the-art. We therefore emphasize the importance of the identification of boundary conditions in liver deformation simulation for future research. Learning-based methods are also attractive for this problem [6]. However, they raise the problem of collecting enough data, which are known to be scarce. 
Impact of the proposed method. The proposed method breaks the initial liver registration problem down into the deformation simulation and pose estimation subproblems. This has two important impacts. First, the proposed method is robust to initialization far from the solution. Second, it allows one to compensate the gravity-load in monocular laparoscopic liver registration in the de facto OR conditions for the first time. The gravity-load has not been compensated by previous liver registration methods, because it is not possible to know the gravity direction in the laparoscope's coordinate frame when both the deformation and pose are optimized simultaneously.

Integration into the standard OR. The proposed method is a semi-automatic solution. It requires one to perform two manual tasks during surgery: (i) measuring the surgery table's tilt angle and (ii) outlining the liver's contour on the laparoscopy image. Once the two tasks are performed, the method has all the inputs it needs to produce a solution automatically in 45 seconds. It is possible to perform the manual tasks in the surgical work flow relatively fast. Task ( $i$ ) can be performed by an assistant using either a smartphone or an accelerometer once the patient's position is fixed at the very beginning of surgery. Therefore it is completely unobtrusive to the surgeon. Task (ii) requires an assistant under the surgeon's supervision to interact with a screen using either a mouse or, if the screen is tactile, their finger. In the mouse case, the assistant clicks a sparse set of points on the liver's partly visible and smooth contours to mark them in the image. Such contours on the laparoscopy images are observable in Figures 3, 8 and 10. For instance, the liver's visible upper silhouette requires clicking about 10 points. The ridge contour also requires clicking about 10 points. The falciform ligament requires clicking only 2 to 3 points and sometimes may not be visible. It takes the assistant about 30 seconds on average to mark the liver's contours by clicking in the laparoscopy image. In the tactile screen case, the surgeon sweeps their finger on the liver's contours to mark them in the laparoscopy image. This is usually a lot faster than clicking. Overall the surgeon spends less than 2 minutes to produce an augmented laparoscopy image. The proposed method has the potential to speed up surgery by revealing the internal anatomy of the liver before resection takes place.

\section{Conclusion}

We have proposed to formulate the initial liver registration problem in monocular laparoscopy as two well-posed successive subproblems: deformation simulation in the preoperative step and pose computation in the intraoperative step. Consequently, we have been able to provide a better constrained and faster solution compared to the state-of-the-art. This is the first solution which compensates the gravity load in liver registration. Contrary to others, the proposed solution can be adopted directly in the de facto OR conditions. As future research, we shall (i) study the boundary conditions for the forward-simulation algorithm, (ii) push further the in-vivo quantitative evaluation of registration, (iii) improve registration by iterating between the deformation simulation and pose computation stages and updating the boundary conditions in order to minimize contour overlap errors on the laparoscopy image, and (iv) explore deep learning approaches to automate contour marking.

Conflict of Interest: The authors declare that they have no conflict of interest.

Ethical approval: All procedures involving human participants were in accordance with the ethical standards of the institutional and/or national research committee and with the 1964 Helsinki declaration and its later amendments or comparable ethical standards.

Informed consent: Informed consent was obtained from the patients included in the study. 


\section{References}

1. Wolf, I., Vetter, M., Wegner, I., Nolden, M., Bottger, T., Hastenteufel, M., Schobinger, M., Kunert, T., Meinzer, H.P.: The medical imaging interaction toolkit (MITK). http://www.mitk.org.

2. Nicolau, S., Soler, L., Mutter, D., Marescaux, J.: Augmented reality in laparoscopic surgical oncology. Surgical Oncology, Volume 20, No. 3, pages 189-201, (2011)

3. Bernhardt, S., Nicolau, S.A., Soler, L., Doignon, C.: The status of augmented reality in laparoscopic surgery as of 2016. Medical Imaging Analysis, Volume 37, pages 66-90 (2017)

4. Koo, B., Ozgur, E., Le Roy, B., Buc, E., Bartoli, A.: Deformable registration of a preoperative 3D liver volume to a laparoscopy image using contour and shading cues. MICCAI, (2017)

5. Adagolodjo, Y., Trivisonne, R., Haouchine, N., Cotin, S., Courtecuisse, H.: Silhouette-based pose estimation for deformable organs application to surgical augmented reality. IROS, (2017)

6. Johnsen, S.F., Thompson, S., Clarkson, M.J., Modat, M., Song, Y., Totz, J., Gurusamy, K., Davidson, B., Taylor, Z.A., Hawkes, D.J., Ourselin, S.: Database-based estimation of liver deformation under pneumoperitoneum for surgical image-guidance and simulation. MICCAI, (2015)

7. Bano, J., Hostettler, A., Nicolau, S.A., Doignon, C., Wu, H.S., Huang, M.H., Soler, L., Marescaux, J.: Simulation of the abdominal wall and its arteries after pneumoperitoneum for guidance of port positioning in laparoscopic surgery. ISVC, (2012)

8. Nimura, Y., Qu, J.D., Hayashi, Y., Oda, M., Kitasaka, T., Hashizume, M., Misawa, K., Mori, K.: Pneumoperitoneum simulation based on mass-spring-damper models for laparoscopic surgical planning. Journal of Medical Imaging, Volume 2, No. 4, 044004 (2015)

9. Bano, J., Hostettler, A., Nicolau, S.A., Cotin, S., Doignon, C., Wu, H.S., Huang, M.H., Soler, L., Marescaux, J.: Simulation of pneumoperitoneum for laparoscopic surgery planning. MICCAI, (2012)

10. Collins, T., Pizarro, D., Bartoli, A., Bourdel, N., Canis, M.: Computer-aided laparoscopic myomectomy by augmenting the uterus with pre-operative MRI data. ISMAR, (2014)

11. Bernhardt, S., Nicolau, S., Bartoli, A., Agnus, V., Soler, L., Doignon, C.: Using shading to register an intraoperative CT scan to a laparoscopic image. Workshop CARE at MICCAI, (2015)

12. Saito, A., Nakao, M., Uranishi, Y., Matsuda, T.: Deformation estimation of elastic bodies using multiple silhouette images for endoscopic image augmentation. ISMAR, (2015)

13. Haouchine, N., Roy, F., Untereiner, L., Cotin, S.: Using contours as boundary conditions for elastic registration during minimally invasive hepatic surgery. IROS, (2016)

14. Suwelack, S., Röhl, S., Bodenstedt, S., Reichard, D., Dillmann, R., Thiago, S., Maier-Hein, L., Wagner, M., Wünscher, J., Kenngott, H., Müller, BP., Spiedel, S.: Physics-based shape matching for intraoperative image guidance. Medical Physics, Volume 41, Issue 11, (2014)

15. Bender, J., Koschier, D., Charrier, P., Weber, D.: Position-based simulation of continuous materials. Computer \& Graphics, (2014)

16. Toledo, M., Ribeiro, P.C.: Radiological evaluation of a liver simulator in comparison to a human real liver. International Nuclear Atlantic Conference, INAC, (2009)

17. Mourcou, Q., Fleury, A., Franco, C., Klopcic, F., Vuillerme, N.: Performance evaluation of smartphone inertial sensors measurement for range of motion. Sensors, Volume 15, pages 23168-23187 (2015)

18. Whiteley, J.: The solution of inverse non-linear elasticity problems that arise when locating breast tumours. Journal of Theoretical Medicine, Volume 6, No. 3, pages 143-149 (2005)

19. Carter, T., Tanner, C., Beechey-Newman, N., Barratt, D., Hawkes, D.: MR navigated breast surgery: method and initial clinical experience. MICCAI, (2008)

20. Eiben, B., Han, L., Hipwell, J., Mertzanidou, T., Kabus, S., Buelow, T., Lorenz, C., Newstead, G.M., Abe, H., Keshtgar, M., Ourselin, S., Hawkes, D.J.: Biomechanically guided prone-to-supine image registration of breast MRI using an estimated reference state. ISBI, (2013)

21. Sellier, M.: An iterative method for the inverse elasto-static problem. Journal of Fluids and Structures, Volume 27, pages 1461-1470 (2011)

22. Morin, F., Courtecuisse, H., Chabanas, M., Payan, Y.: Rest shape computation for highly deformable model of brain. Computer Methods in Biomechanics and Biomedical Engineering, Volume 18, (2015)

23. Eiben, B., Vavourakis, V., Hipwell, J., Kabus, S., Lorenz, C., Buelow, T., Hawkes, D.J.: Breast deformation modelling: comparison of methods to obtain a patient specific unloaded configuration. Conference on Medical Imaging - Image-Guided Procedures, Robotic Interventions, and Modeling, (2014)

24. Mackling, M., Müller, M., Chentanez, N.: XPBD: Position-based simulation of compliant constrained dynamics. MIG, (2016)

25. Lepetit, V., Moreno-Noguer, F., Fua, P.: EPnP: An accurate O(n) solution to the PnP problem. International Journal of Computer Vision, Volume 81, No. 2, pages 155-166 (2008)

26. Bosman, J., Haouchine, N., Dequidt, J., Peterlik, I.: The role of ligaments: patient-specific or scenariospecific?. ISBMS, (2014) 